\title{
Aarrso Estrutura e processo de trabalho para as ações de alimentação e nutrição na Atenção Primária à Saúde no Brasil, 2014*
}

doi: 10.1590/S1679-49742021000200015

\author{
Structure and work process for food and nutrition actions in Primary Health Care in Brazil, \\ 2014
}

\section{Estructura y proceso de trabajo para las acciones de alimentación y nutrición en la Atención Primaria de Salud en Brasil, 2014}

\author{
Patrícia Maria de Oliveira Machado' - (D orcid.org/0000-0002-8630-8318 \\ Josimari Telino de Lacerda² - (D orcid.org/0000-0002-1992-4030 \\ Claudia Flemming Colussi ${ }^{2}$ - (1) orcid.org/0000-0002-3395-9125 \\ Maria Cristina Marino Calvo² - (D orcid.org/0000-0001-8661-7228 \\ 'Universidade Federal da Integração Latino-Americana, Curso de Graduação em Medicina, Foz do Iguaçu, PR, Brasil \\ ¿Universidade Federal de Santa Catarina, Departamento de Saúde Pública, Florianópolis, SC, Brasil
}

\section{Resumo}

Objetivo: Avaliar as condições de estrutura e de processo de trabalho para as ações de alimentação e nutrição na Atenção Primária à Saúde, no Brasil. Métodos: Estudo transversal, sobre dados secundários do Programa de Melhoria do Acesso e da Qualidade da Atenção Básica. Foram descritas as proporções de adequação das unidades básicas de saúde (UBS) e equipes de saúde segundo variáveis organizacionais, pela razão da prevalência e regressão de Poisson. Resultados: Foram analisadas 19.793 UBS e 24.549 equipes; $35,0 \%(n=6.928)$ das UBS mostraram-se adequadas na estrutura e 7,9\% $(\mathrm{n}=1.934)$ das equipes adequadas ao processo de trabalho. Nas análises de associação, destacaram-se as UBS da região Sul $(44,7 \%)$ e as equipes da região Sudeste (10,9\%), além de municípios com mais de 300 mil habitantes. Conclusão: As UBS e equipes analisadas apresentaram baixas proporções de adequação de estrutura e de processo de trabalho para as ações de alimentação e nutrição.

Palavras-chave: Estrutura dos Serviços; Atenção Primária à Saúde; Programas e Políticas de Nutrição e Alimentação; Estudos Transversais.

\footnotetext{
*Artigo derivado de tese de doutorado intitulada 'Atenção Nutricional no Âmbito da Atenção Primária à Saúde: análise de implantação em municípios brasileiros', defendida por Patrícia Maria de Oliveira Machado junto ao Programa de Pós-Graduação em Saúde Coletiva da Universidade Federal de Santa Catarina em julho de 2018. Patrícia Maria de Oliveira Machado foi bolsista de pós-graduação da Coordenação de Aperfeiçoamento de Pessoal de Nível Superior/Ministério da Educação (Capes/MEC): Processo no 1422415.
}

Endereço para correspondência:

Patrícia Maria de Oliveira Machado - Universidade Federal da Integração Latino-Americana, Instituto Latino-Americano de Ciências da Vida e da Natureza, Jardim Universitário, Loteamento Universitário das Américas, Av. Tarquínio Joslin dos Santos, $n^{\circ}$ 1000, sala G201, Foz do Iguaçu, PR, Brasil. CEP: 85870-650

E-mail: patriciamomachado@gmail.com 


\section{Introdução}

A alimentação inadequada é importante fator de risco para os agravos em saúde de maior morbimortalidade, como as doenças crônicas não transmissíveis (DCNT).$^{1,2}$ Este quadro também se caracteriza pela coexistência de agravos agudos não solucionados, como desnutrição, doenças infecciosas e carências nutricionais, sendo a anemia ferropriva uma das mais prevalentes. ${ }^{3}$ Nesse sentido, uma agenda única para as ações de alimentação e nutrição constitui importante desafio para o Sistema Único de Saúde (SUS), principalmente na Atenção Primária à Saúde (APS). ${ }^{4}$

A APS é reconhecida como locus ideal para 0 desenvolvimento de ações de alimentação e nutrição, mesmo em países que não possuem modelos de atenção à saúde pautados pela organização de sua atenção primária, ou de abrangência universal. Os cuidados em saúde no nível comunitário (community-based care) identificam-se como o espaço mais efetivo para a promoção de hábitos alimentares adequados e saudáveis, além da prevenção das DCNT e agravos nutricionais. 4,5

\section{Uma agenda única para as ações de alimentação e nutrição constitui importante desafio para o Sistema Único de Saúde, principalmente na Atenção Primária à Saúde.}

A organização das ações de nutrição na APS tem como propósito melhorar as condições de alimentação da população, por meio de estratégias que promovam (i) as práticas alimentares adequadas, organizem (ii) o processo de trabalho em saúde para o cuidado integral, e implantem (iii) o monitoramento contínuo do estado alimentar e nutricional com classificação de risco nutricional, prevenção e controle dos agravos associados à alimentação, em todos os ciclos de vida. ${ }^{4,5}$

A capilaridade da APS e a expansão das equipes multiprofissionais contribuíram para ampliar 0 acesso à saúde, esta que também depende de contínua qualificação do processo de trabalho das equipes e estruturação das unidades básicas de saúde (UBS). As UBS são essenciais para a estruturação de um modelo assistencial integral, voltado ao planejamento territorial com base nos fatores determinantes de saúde, no quadro demográfico e epidemiológico da população de abrangência, utilizando a estratificação de risco para o funcionamento dos sistemas em redes. ${ }^{6,7}$

Identificado como um dos desafios da consolidação das ações de alimentação e nutrição na APS, o Sistema de Vigilância Alimentar e Nutricional (SISVAN) necessita de condições adequadas de estrutura e processo de trabalho para se desenvolver, monitorar e exercer o esperado controle sobre o quadro epidemiológico atual, em cada localidade do país. ${ }^{7,8}$ Os procedimentos sistemáticos de inserção de dados nos sistemas de informações, a implementação de programas para 0 enfrentamento das deficiências de micronutrientes, a promoção da alimentação saudável e a prevenção das doenças crônicas com foco de atenção na obesidade são ações a serem executadas, enquanto parte do processo de trabalho das equipes de saúde da APS..$^{8-10}$

O Brasil adotou iniciativas para avaliar as condições de acesso e qualidade da APS, por meio de políticas indutoras como o Programa Nacional de Melhoria do Acesso e da Qualidade da Atenção Básica (PMAQ-AB), conduzido pelo Ministério da Saúde. 0 segundo ciclo do PMAQ-AB, implementado entre os anos de $2013 \mathrm{e}$ 2014, avaliou 30.523 equipes de saúde e 24.055 UBS. ${ }^{11}$

0 objetivo da presente pesquisa foi avaliar as condições de estrutura e processo de trabalho para o desenvolvimento das ações de alimentação e nutrição pelas unidades e equipes de saúde da APS participantes do segundo ciclo do PMAQ-AB no Brasil.

\section{Métodos}

Trata-se de um estudo transversal com dados do PMAQ-AB, disponíveis no sítio eletrônico do Departamento de Atenção Básica do Ministério da Saúde (DAB/MS).

O PMAQ-AB consistiu em uma política indutora, implementada a partir de 2011, que elevou os repasses federais aos municípios participantes mediante o cumprimento de padrões de qualidade definidos para os processos de gestão e assistência à saúde na APS. ${ }^{11} 0$ programa contava com fases de adesão e contratualização, desenvolvimento, avaliação externa e recontratualização, sendo a avaliação externa a fase de coleta de dados junto às UBS e equipes de saúde em todo o país. ${ }^{11}$

Como critérios de inclusão no estudo, foram consideradas as equipes e unidades de saúde com todas as informações disponíveis nos módulos I e II do PMAQ-AB, 
localizadas em municípios que aderiram ao programa com pelo menos $80 \%$ do total das equipes cadastradas no Cadastro Nacional de Estabelecimentos de Saúde (CNES), para se evitar viés de seleção das equipes.

0 desfecho primário do estudo foi a adequação da estrutura para as UBS e a adequação do processo de trabalho para as equipes de saúde (adequado; inadequado). Foram incluídas variáveis sobre estrutura, processo de trabalho e características organizacionais da UBS:

a) Adequação de estrutura

- Balança antropométrica-adulto, para até $150 \mathrm{~kg}$ ou 200kg (sim; não);

- Balança infantil (sim; não);

- Régua antropométrica (sim; não);

- Caderneta de Saúde, da criança e da gestante ( $\operatorname{sim} ;$ não);

- Fita métrica (sim; não);

- Sais para reidratação oral (sim; não);

- Sulfato ferroso (sim; não);

- Ácido fólico (sim; não).

b) Adequação de processo de trabalho

- Utilização de sistema de registro de informações (sim; não);

- Oferta de ações e consultas para usuários com obesidade (sim; não);

- Utilização de protocolo de estratificação de risco para crescimento e desenvolvimento de menores de 2 anos (sim; não);

- Programação de ações de acordo com o risco classificado (sim; não);

- Registro de usuários com obesidade encaminhados a outros pontos de atenção (sim; não);

- Busca ativa das crianças menores de 2 anos em desenvolvimento (sim; não);

- Busca ativa das crianças menores de 2 anos com baixo peso (sim; não);

- Oferta de exames pela rede de serviços, tais como creatinina, perfil lipídico, hemoglobina glicosilada, hematócrito e hemoglobina (sim; não);

- Realização de ações de vigilância alimentar e nutricional (sim; não);

- Monitoramento de índices de aleitamento materno e alimentação complementar saudável (sim; não);

- Conhecimento da Norma Brasileira de Comercialização de Alimentos para Lactentes e Crianças de Primeira Infância (NBCAL) (sim; não);
- Mensuração de peso e altura de menores de 2 anos, gestantes, adultos com diabetes mellitus e hipertensão, usuários atendidos na unidade de saúde (sim; não);

- Oferta de ações educativas e de promoção da saúde sobre alimentação saudável e aleitamento materno (sim; não);

- Desenvolvimento de ações de atividade física na UBS (sim; não).

c) Variáveis independentes

- UBS com equipes mínimas completas (sim; não) a partir da adequação do número de equipes e número de profissionais disponíveis;

- Apoio de equipe de Saúde Bucal (sim; não);

- Apoio de nutricionista (sim; não);

- Apoio dos Núcleos de Apoio à Saúde da Família (NASF) (sim; não);

- População descoberta na área de abrangência (sim; não).

Ademais, foram acrescentadas as variáveis 'região' do país (Nordeste, Norte, Centro-Oeste, Sudeste e Sul) e 'porte municipal' (em habitantes: até $10.000 ; 10.001$ a $30.000 ; 30.001$ a 100.000 ; 100.001 a 300.000; mais de 300.000).

Para análise de estrutura das UBS, considerou-se a disponibilidade dos materiais ou insumos a partir do módulo I do PMAQ-AB, e para a análise do processo de trabalho, a resposta positiva das equipes para as variáveis constantes no módulo II do PMAQ. Foram consideradas adequadas as unidades e equipes que apresentaram 100\% dos itens analisados.

Além de dados do PMAQ-AB para estrutura e processo de trabalho, foram utilizados dados do CNES, para ciência do número de equipes existentes nos municípios e cálculo do percentual de adesão como critério de inclusão; e dados do Instituto Brasileiro de Geografia e Estatística (IBGE), para classificação da região do país e número de habitantes. Selecionou-se o período de 2014 como referência para todas as coletas, por se tratar do ano de encerramento das coletas do PMAQ-AB e permitir a correspondência temporal entre os diferentes bancos de dados.

A adequação de estrutura e do processo de trabalho segundo as características organizacionais foi estimada pela prevalência e intervalo de confiança de $95 \%\left(\mathrm{IC}_{95 \%}\right)$ na análise descritiva, e pela razão 
da prevalência $(\mathrm{RP})$ e $\mathrm{IC}_{95 \%}$ nas análises bruta e ajustada utilizando-se regressão de Poisson, com p-valor obtido pelo teste de Wald.

Os potenciais fatores de confusão foram controlados com regressão de Poisson, indicada para estudos de corte transversal com prevalência para variáveis de desfecho maiores que $20 \% .^{12}$ As variáveis independentes foram inseridas no modelo de maneira crescente, conforme sua significância estatística, tendo permanecido na análise caso mantivessem significância estatística $(\mathrm{p}<0,05)$. Para a análise de adequação do processo de trabalho, foi inserida, nos modelos bruto e ajustado, a variável 'adequação de estrutura' (inadequada; adequada) como variável independente. Os dados foram processados com uso do software Microsoft Office Excel® (Microsoft Corporation, Estados Unidos) e transferidos para 0 software Stata ${ }^{\circledR} 12.0$ (Stata Corporation, College Station, TX, Estados Unidos).

Os dados, provenientes das diferentes fontes de informações, foram coletados dos respectivos sítios eletrônicos no formato $c s v$ e, posteriormente, transformados em arquivos do programa Excel ${ }^{\circledR}$. Eles foram agrupados em dois bancos distintos, um para análise da estrutura das UBS e outro para análise do processo de trabalho. A variável utilizada para o pareamento dos registros nos bancos de dados foi o código do município no IBGE. Procedeu-se à limpeza dos dados classificados como missing values ou erros identificados com numeração inexistente nos dicionários de coleta de dados do PMAQ-AB, também disponíveis nos microdados do Ministério da Saúde. ${ }^{11}$

0 projeto da pesquisa foi aprovado pelo Comitê de Ética em Pesquisas com Seres Humanos da Universidade Federal de Santa Catarina (CEPSH/UFSC): Parecer $\mathrm{n}^{0}$ 2.047.153, emitido em 28 de agosto de 2017; Certificado de Apresentação de Apreciação Ética (CAAE) $n^{0}$ 63164416.5.0000.0121.

\section{Resultados}

A amostra analisada contemplou 19.793 unidades de saúde e 24.549 equipes de saúde, distribuídas em 3.903 municípios de 26 estados brasileiros. Não fizeram parte da amostra as equipes e unidades de saúde localizadas no Distrito Federal, Unidade da Federação onde a adesão das equipes de saúde ao PMAQ foi de $46 \%$.
Das UBS analisadas em todo o país, 35\% foram consideradas adequadas quanto à estrutura disponível para as ações de alimentação e nutrição $(n=6.928)$. As UBS localizadas na região Sul $(44,7 \%)$, instaladas em municípios com mais de 300 mil habitantes (42,1\%), dotadas de equipe de Saúde Bucal (36,2\%), que contavam com apoio do nutricionista (39,2\%) e do $\operatorname{NASF}(37,7 \%)$ apresentaram maior adequação de estrutura (Tabela 1).

Entre as equipes de saúde, 7,9\% ( $\mathrm{n}=1.934)$ foram consideradas adequadas quanto ao processo de trabalho para as ações de alimentação e nutrição. Destacaram-se as equipes de saúde da região Sudeste $(10,9 \%)$, em municípios com mais de 300 mil habitantes $(12,9 \%)$, apoiadas por nutricionista $(10,2 \%)$ e por NASF $(9,6 \%)$ (Tabela 1$)$.

Na Figura 1, apresenta-se a disponibilidade de materiais para estrutura das ações de alimentação e nutrição nas UBS. Nenhum dos itens analisados atingiu $100 \%$ de disponibilidade nas unidades de saúde no país, sendo os de maior frequência a fita métrica (96,6\%), a balança-adulto ( $95,7 \%)$ e a balança infantil $(92,9 \%)$. Os itens com menor disponibilidade foram a Caderneta de Saúde da Criança $(71,4 \%)$, sais para reidratação oral $(87,0 \%)$ e a régua antropométrica $(88,9 \%)$ (Figura 1).

Das 21 ações de alimentação e nutrição analisadas para o processo de trabalho das equipes, oito eram realizadas por mais de $90 \%$ das equipes (Figura 2 ). Entre essas oito, destacaram-se a oferta de exames laboratoriais na rede de serviços, com variação entre 92,1 e $97,5 \%$, e a mensuração de peso e altura de gestantes e de crianças menores de 2 anos $(95,9 \%)$. 0 registro de usuários com obesidade encaminhados a outros pontos de atenção da rede assistencial foi realizado por 39\% das equipes, e a programação de ações conforme a estratificação de risco foi a ação com menor frequência entre as equipes de saúde analisadas (35,8\%) (Figura 2).

Pernambuco, Ceará e Santa Catarina apresentaram as maiores frequências de UBS adequadas quanto à estrutura: $53,2 \%, 49,3 \%$ e $49,2 \%$, respectivamente. Na adequação do processo de trabalho, destacaram-se as equipes dos estados do Ceará (15,8\%), São Paulo $(14,8 \%)$ e Rio de Janeiro (10,4\%). Nenhuma das equipes avaliadas nos estados do Acre, Rondônia e Amapá teve seu processo de trabalho considerado adequado para as ações de alimentação e nutrição (Figura 3). 
Tabela 1 - Distribuição das unidades básicas de saúde e equipes de saúde participantes da avaliação externa do $2^{\circ}$ ciclo do PMAQ-AB, ${ }^{\text {B Brasil, }} 2014$

\begin{tabular}{|c|c|c|c|c|c|c|}
\hline \multirow{3}{*}{ Variáveis } & \multicolumn{3}{|c|}{ Unidades básicas de saúde } & \multicolumn{3}{|c|}{ Equipes de saúde } \\
\hline & \multirow{2}{*}{$\begin{array}{l}\text { Total } \\
\text { n (\%) }\end{array}$} & \multicolumn{2}{|c|}{ Estrutura adequada } & \multirow{2}{*}{$\begin{array}{l}\text { Total } \\
\text { n (\%) }\end{array}$} & \multicolumn{2}{|c|}{$\begin{array}{c}\text { Processo de trabalho } \\
\text { adequado }\end{array}$} \\
\hline & & $\mathrm{n}$ & $\%\left(\mathrm{IC}_{95 \%}^{\mathrm{b}}\right)$ & & $\mathrm{n}$ & $\%\left(\mathrm{IC}_{95 \%}{ }^{\mathrm{b}}\right)$ \\
\hline Brasil & $19.793(100,0)$ & 6.928 & $35,0(34,3 ; 35,7)$ & $24.549(100,0)$ & 1.934 & $7,9(7,5 ; 8,2)$ \\
\hline \multicolumn{7}{|l|}{ Região } \\
\hline Norte & $1.207(6,1)$ & 269 & $22,3(20,0 ; 24,7)$ & $1.519(6,2)$ & 47 & $4,0(2,3 ; 4,1)$ \\
\hline Nordeste & $7.845(39,6)$ & 3.019 & $38,5(37,4 ; 39,6)$ & $8.573(34,9)$ & 555 & $6,5(6,0 ; 7,0)$ \\
\hline Sudeste & $5.967(30,2$ & 1.837 & $30,8(29,6 ; 32,0)$ & $8.606(35,1)$ & 939 & $10,9(10,3 ; 11,6)$ \\
\hline Sul & $3.105(15,7)$ & 1.387 & $44,7(42,9 ; 46,4)$ & $3.882(15,8)$ & 304 & $7,8(7,0 ; 8,7)$ \\
\hline Centro-0este & $1.669(8,4)$ & 416 & $24,9(22,9 ; 27,1)$ & $1.969(8,0)$ & 89 & $4,5(3,7 ; 5,5)$ \\
\hline \multicolumn{7}{|c|}{ Porte municipal (em habitantes) } \\
\hline Até 10.000 & $3.280(16,6)$ & 1.114 & $34,0(32,4 ; 35,6)$ & $3.664(14,9)$ & 202 & $5,5(4,8 ; 6,3)$ \\
\hline 10.001 a 30.000 & $6.833(34,5)$ & 2.154 & $31,5(30,4 ; 32,6)$ & $7.222(29,4)$ & 431 & $6,0(5,4 ; 6,5)$ \\
\hline 30.001 a 100.000 & $4.983(25,2)$ & 1.763 & $35,4(34,1 ; 36,7)$ & $5.439(22,2)$ & 367 & $6,8(6,1 ; 7,4)$ \\
\hline 100.001 a 300.000 & $2.276(11,5)$ & 878 & $38,6(36,6 ; 40,6)$ & $3.010(12,3)$ & 260 & $8,6(7,7 ; 9,6)$ \\
\hline Mais de 300.000 & $2.421(12,2)$ & 1.019 & $42,1(40,1 ; 44,1)$ & $5.214(21,4)$ & 674 & $12,9(12,0 ; 13,9)$ \\
\hline \multicolumn{7}{|c|}{ Equipe de Saúde Bucal } \\
\hline Não & $4.106(20,7)$ & 1.246 & $30,4(29,0 ; 31,8)$ & $8.131(33,1)$ & 710 & $8,7(8,1 ; 9,4)$ \\
\hline Sim & $15.687(79,3)$ & 5.682 & $36,2(35,5 ; 37,0)$ & $16.418(66,9)$ & 1.224 & $7,5(7,1 ; 7,9)$ \\
\hline \multicolumn{7}{|c|}{ Equipes mínimas completas } \\
\hline Não & $3.815(19,3)$ & 1.317 & $34,5(33,0 ; 36,0)$ & $-^{c}$ & $-^{c}$ & $-^{c}$ \\
\hline Sim & $15.978(80,7)$ & 5.611 & $35,1(34,4 ; 35,9)$ & & & \\
\hline \multicolumn{7}{|l|}{ Nutricionista } \\
\hline Não & $10.637(53,7)$ & 3.339 & $31,4(30,5 ; 32,3)$ & $12.737(51,9)$ & 724 & $5,7(5,3 ; 6,1)$ \\
\hline Sim & $9.156(46,3)$ & 3.589 & $39,2(38,2 ; 40,2)$ & $11.812(48,1)$ & 1.210 & $10,2(9,7 ; 10,8)$ \\
\hline \multicolumn{7}{|c|}{ Núcleo de Apoio à Saúde da Família (NASF) } \\
\hline Não & $8.501(43,0)$ & 2.675 & $31,5(30,5 ; 32,5)$ & $10.053(40,9)$ & 549 & $5,5(5,0 ; 5,9)$ \\
\hline Sim & $11.292(57,0)$ & 4.253 & $37,7(36,8 ; 38,6)$ & $14.496(59,1)$ & 1.385 & $9,6(9,1 ; 10,0)$ \\
\hline \multicolumn{7}{|c|}{ População descoberta na área de abrangência } \\
\hline Sim & $5.871(33,9)$ & 1.894 & $32,3(31,1 ; 33,5)$ & $7.443(34,1)$ & 450 & $6,1(5,5 ; 6,6)$ \\
\hline Não & $11.433(66,1)$ & 4.433 & $38,8(37,9 ; 39,7)$ & $14.352(65,9)$ & 1.426 & $9,9(9,4 ; 10,4)$ \\
\hline
\end{tabular}

a) PMAQ-AB: Programa de Melhoria do Acesso e da Qualidade da Atenção Básica; b) IC $95 \%$ : intervalo de confiança de $95 \%$; c) Informações não disponíveis no módulo II do PMAQ-AB $2^{\circ}$ ciclo para classificação das equipes.

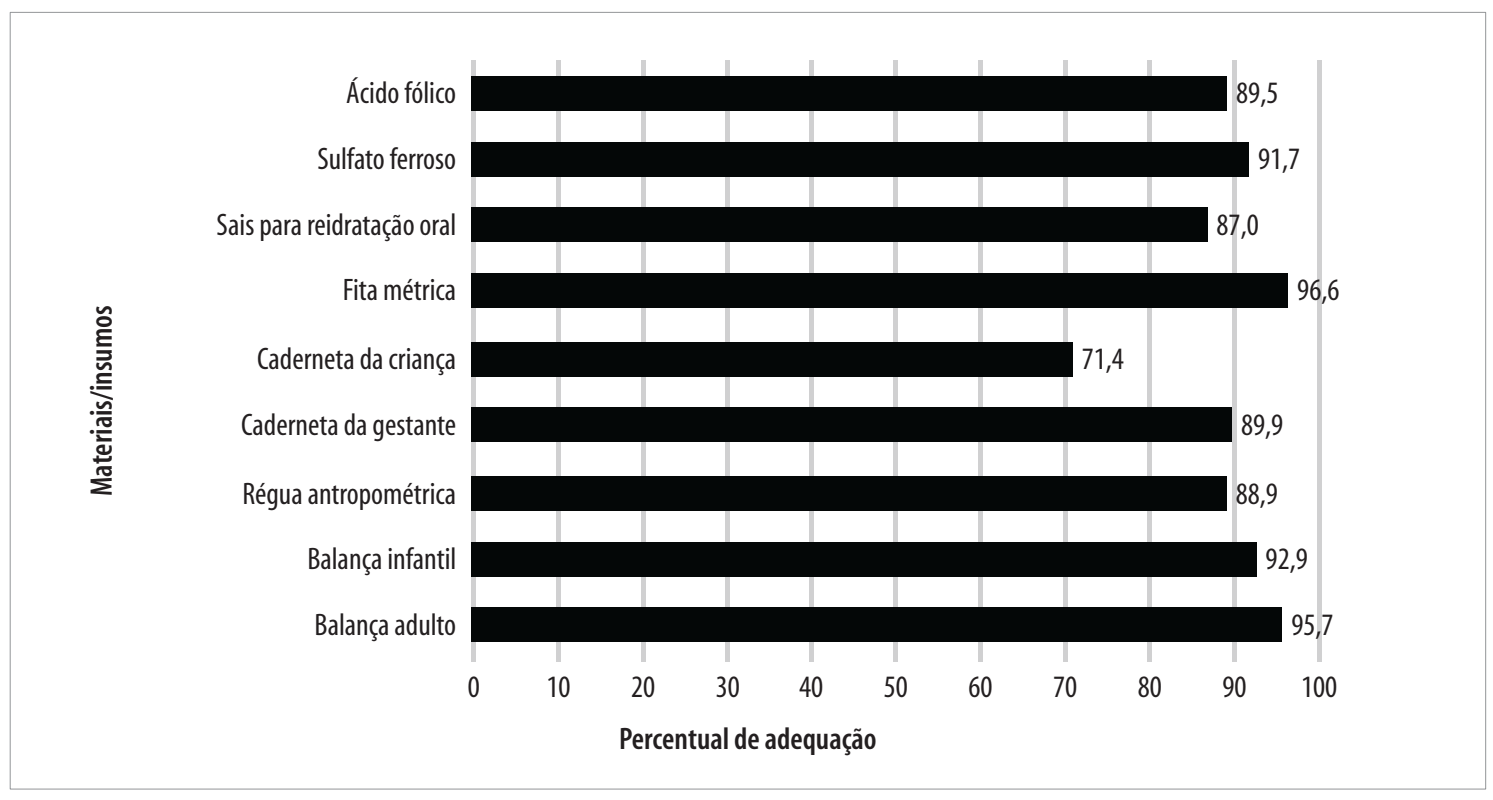

a) UBS: unidade básica de saúde; b) PMAQ-AB:Programa de Melhoria do Acesso e da Qualidade da Atenção Básica.

Figura 1 - Distribuição percentual da disponibilidade de insumos/equipamentos que compõem a estrutura para as ações de alimentação e nutrição nas UBS, ${ }^{\mathrm{a}}$ de acordo com $02^{\circ}$ ciclo do PMAQ-AB, ${ }^{\mathrm{b}}$ Brasil, 2014 


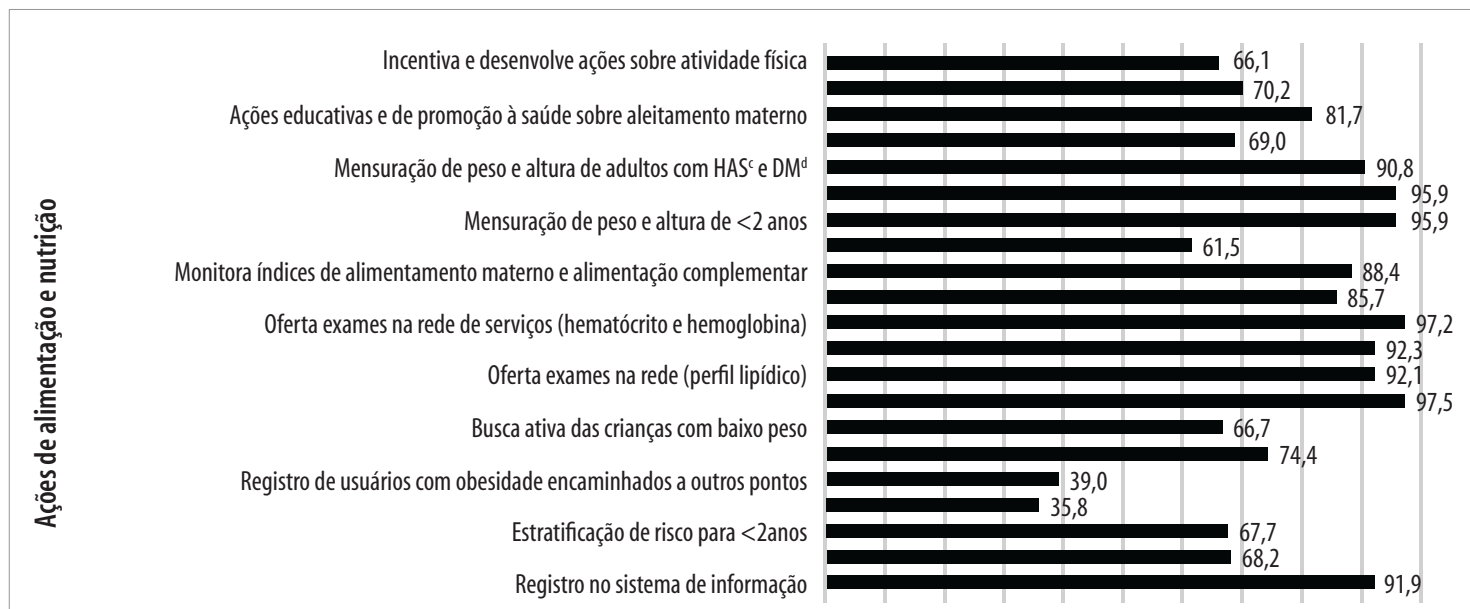

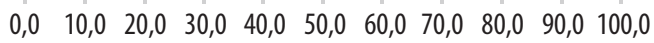

Percentual de adequação

a) UBS: unidade básica de saúde; b) PMAQ-AB:Programa de Melhoria do Acesso e da Qualidade da Atenção Básica;c) HAS: hipertensão arterial sistêmica; d) DM: diabetes melittus.

\section{Figura 2 - Distribuição percentual das ações de alimentação e nutrição que compõem o processo de trabalho das equipes das UBS ${ }^{a}$ na Atenção Primária à Saúde, de acordo com $02^{\circ}$ ciclo do PMAQ-AB, ${ }^{b}$ Brasil, 2014}

Tabela 2 - Análises bruta e ajustada da adequação de estrutura e adequação do processo de trabalho para as ações de alimentação e nutrição na Atenção Primária à Saúde, de acordo com $02^{\circ}$ ciclo do PMAQ-AB, Brasil, 2014

\begin{tabular}{|c|c|c|c|c|c|c|c|c|}
\hline \multirow[b]{2}{*}{ Variáveis } & \multicolumn{4}{|c|}{ Adequação de estrutura } & \multicolumn{4}{|c|}{ Adequação do processo de trabalho } \\
\hline & $\begin{array}{c}\mathrm{RPb}^{\mathrm{B}}\left(\mathrm{IC}_{95 \%} \mathrm{C}\right) \\
\text { bruta }\end{array}$ & p-valor ${ }^{d}$ & $\begin{array}{l}\mathrm{RPb}^{\mathrm{b}}\left(\mathrm{IC}_{\left.\mathrm{C}_{95 \%}{ }^{\mathrm{C}}\right)}\right. \\
\text { ajustada }\end{array}$ & $p^{d}$ & $\begin{array}{c}\mathrm{RPb}^{\mathrm{b}}\left(\mathrm{IC}_{95 \%} \mathrm{C}\right) \\
\text { bruta }\end{array}$ & $p^{d}$ & $\begin{array}{l}\mathrm{RPb}^{\mathrm{b}}\left(\mathrm{IC}_{95 \%}{ }^{\mathrm{C}}\right) \\
\text { ajustada }{ }^{1}\end{array}$ & p-valor \\
\hline \multicolumn{9}{|l|}{ Região } \\
\hline Norte & 1,00 & $<0,001$ & 1,00 & $<0,001$ & 1,00 & $<0,001$ & 1,00 & $<0,001$ \\
\hline Nordeste & $1,72(1,55 ; 1,93)$ & & $1,76(1,53 ; 2,02)$ & & $2,09(1,56 ; 2,80)$ & & $1,48(1,10 ; 2,01)$ & \\
\hline Sudeste & $1,38(1,25 ; 1,55)$ & & $1,31(1,14 ; 1,51)$ & & $3,53(2,64 ; 4,70)$ & & $2,08(1,54 ; 2,80)$ & \\
\hline Sul & $2,00(1,79 ; 2,24)$ & & $1,91(1,65 ; 2,20)$ & & $2,53(1,87 ; 3,42)$ & & $1,61(1,18 ; 2,20)$ & \\
\hline Centro-0este & $1,12(0,98 ; 1,28)$ & & $1,15(0,97 ; 1,36)$ & & $1,46(1,03 ; 2,07)$ & & $1,28(0,89 ; 1,82)$ & \\
\hline \multicolumn{9}{|c|}{ Porte municipal (em babitantes) } \\
\hline Até 10.000 & 1,00 & $<0,001$ & 1,00 & & 1,00 & $<0,001$ & 1,00 & \\
\hline 10.001 a 30.000 & $0,93(0,87 ; 0,98)$ & & $0,91(0,84 ; 0,98)$ & & $1,08(092 ; 1,27)$ & & $1,15(0,96 ; 1,37)$ & \\
\hline 30.001 a 100.000 & $1,04(0,98 ; 1,11)$ & & $1,04(0,95 ; 1,13)$ & & $1,22(1,04 ; 1,45)$ & & $1,21(1,01 ; 1,46)$ & \\
\hline 100.001 a 300.000 & $1,14(1,06 ; 1,22)$ & & $1,19(1,08 ; 1,31)$ & & $1,57(1,31 ; 1,87)$ & & $1,48(1,22 ; 1,81)$ & \\
\hline Mais de 300.000 & $1,24(1,16 ; 1,32)$ & & $1,29(1,18 ; 1,41)$ & & $2,34(2,02 ; 5,73)$ & & $1,75(1,46 ; 2,09)$ & \\
\hline \multicolumn{9}{|c|}{ Equipe de Saúde Bucal } \\
\hline Não & 1,00 & $<0,001$ & 1,00 & & 1,00 & $<0,001$ & 1,00 & \\
\hline Sim & $1,19(1,13 ; 1,26)$ & & $1,18(1,10 ; 1,26)$ & & $0,85(0,78 ; 0,93)$ & & $1,07(0,97 ; 1,19)$ & \\
\hline \multicolumn{9}{|c|}{ Equipes mínimas completas } \\
\hline Não & 1,00 & 0,489 & - & & $-{ }^{d}$ & & $-{ }^{\mathrm{d}}$ & \\
\hline Sim & $1,02(0,97 ; 1,07)$ & & & & & & & \\
\hline \multicolumn{9}{|l|}{ Nutricionista } \\
\hline Não & 1,00 & $<0,001$ & 1,00 & & 1,00 & $<0,001$ & 1,00 & \\
\hline Sim & $1,25(1,20 ; 1,30)$ & & $1,13(1,06 ; 1,20)$ & & $1,80(1,64 ; 1,97)$ & & $1,31(1,17 ; 1,47)$ & \\
\hline \multicolumn{9}{|c|}{ Núcleo de Apoio à Saúde da Família (NASF) } \\
\hline Não & 1,00 & $<0,001$ & 1,00 & & 1,00 & $<0,001$ & 1,00 & \\
\hline Sim & $1,20(1,15 ; 1,24)$ & & $1,03(0,97 ; 1,10)$ & & $1,75(1,59 ; 1,92)$ & & $1,22(1,07 ; 1,38)$ & \\
\hline \multicolumn{9}{|c|}{ População descoberta na área de abrangência } \\
\hline Sim & 1,00 & $<0,001$ & 1,00 & & 1,00 & $<0,001$ & 1,00 & \\
\hline Não & $1,20(1,15 ; 1,26)$ & & $1,19(1,13 ; 1,26)$ & & $1,64(1,48 ; 1,82)$ & & $1,52(1,36 ; 1,69)$ & \\
\hline \multicolumn{9}{|c|}{ Adequação de estrutura } \\
\hline Inadequada & - & - & - & & 1,00 & $<0,001$ & 1,00 & \\
\hline Adequada & & & & & $2,09(1,92 ; 2,28)$ & & $1,70(1,55 ; 1,87)$ & \\
\hline
\end{tabular}

a) PMAQ-AB: Programa de Melhoria do Acesso e da Qualidade da Atenção Básica; b) IC $\mathrm{C}_{95 \%}$ : intervalo de confiança de $95 \%$; $c$ ) Informaçōes não disponíveis no módulo II do PMAQ-AB $2^{\circ}$ ciclo para classificação das equipes; d) Teste de Wald. 


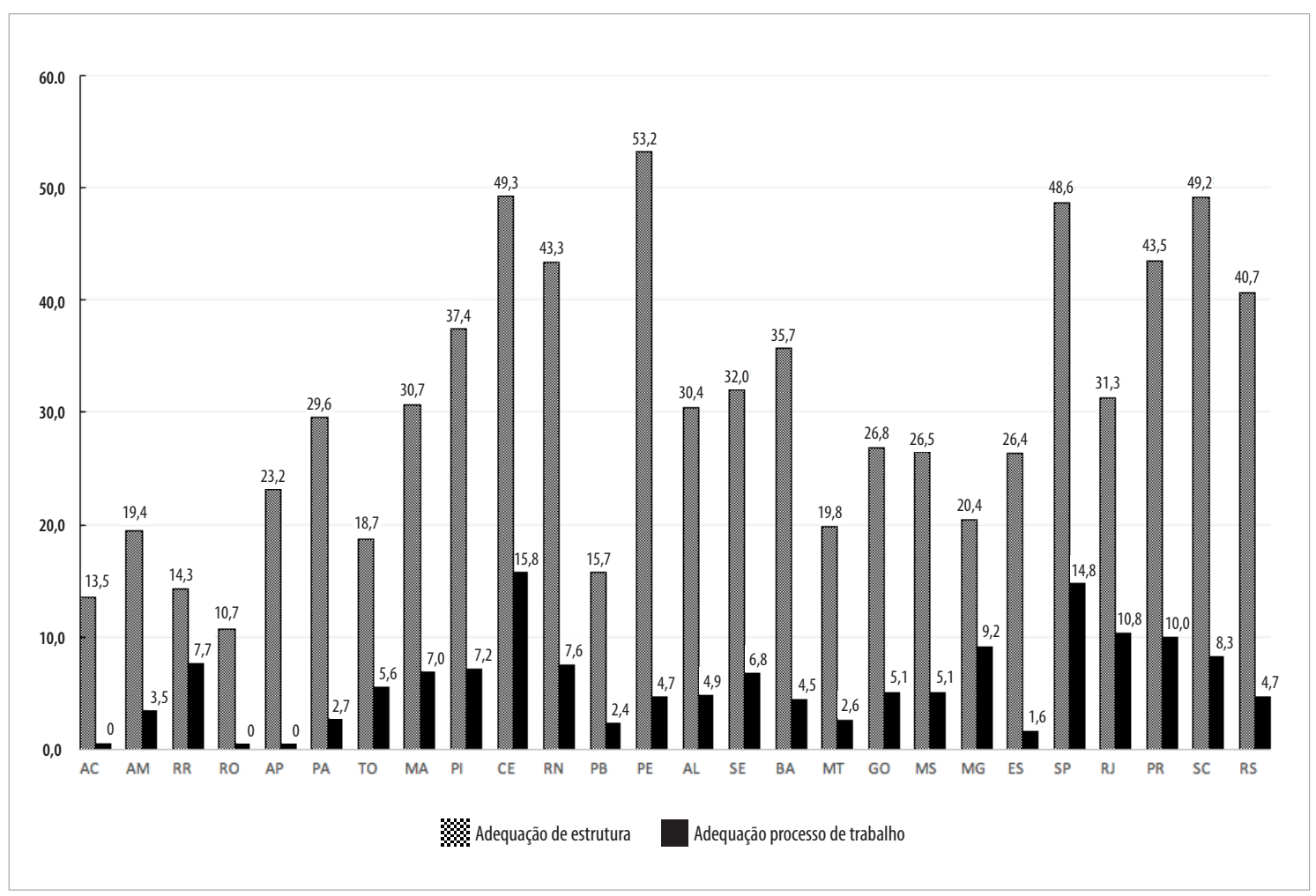

a) PMAQ-AB:Programa de Melhoria do Acesso e da Qualidade da Atenção Básica.

\section{Figura 3 - Percentual de unidades básicas de saúde e equipes de saúde adequadas quanto à estrutura e processo de trabalho para as ações de alimentação e nutrição, segundo Unidades da Federação

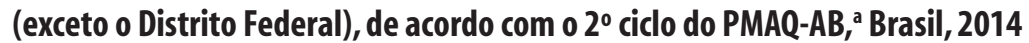

A Tabela 2 apresenta as análises bruta e ajustada, para a associação de adequação de estrutura e adequação do processo de trabalho com as características organizacionais. A região Sul apresentou duas vezes maior probabilidade de adequação de estrutura para as ações de alimentação e nutrição $\left(\mathrm{RP}=2,00-\mathrm{IC}_{95 \%} 1,79 ; 2,24\right)$, seguida das UBS da região Nordeste com maior frequência $(\mathrm{RP}=1,76$ - $\left.\mathrm{IC}_{95 \%} 1,53 ; 2,02\right)$, quando comparadas às UBS da região Norte (Tabela 2 ).

A adequação de estrutura foi $24 \%$ maior para as UBS localizadas em municípios com mais de 300 mil habitantes $\left(\mathrm{RP}=1,24-\mathrm{IC}_{95 \%} 1,16 ; 1,32\right)$, que contavam com apoio de equipe de Saúde Bucal $\left(\mathrm{RP}=1,19-\mathrm{IC}_{95 \%} 1,13 ; 1,26\right)$, de nutricionista $\left(\mathrm{RP}=1,25-\mathrm{IC}_{95 \%} 1,20 ; 1,30\right)$ e de NASF $(\mathrm{RP}=1,20$ - $\left.\mathrm{IC}_{95 \%} 1,15 ; 1,24\right)$ (Tabela 2).

Quanto à adequação do processo de trabalho, as regiões Sudeste $\left(\mathrm{RP}=3,53-\mathrm{IC}_{95 \%} 2,64 ; 4,70\right)$ e Sul $\left(\mathrm{RP}=2,53-\mathrm{IC}_{95 \%} 1,87 ; 3,42\right)$ apresentaram maior percentual de equipes com processo de trabalho adequado. Destacou-se maior adequação do processo de trabalho nas equipes localizadas em municípios de maior porte $\left(\mathrm{RP}=2,34-\mathrm{IC}_{95 \%} 2,02 ; 5,73\right)$, nas equipes apoiadas pelo NASF $\left(\mathrm{RP}=1,75-\mathrm{IC}_{95 \%}\right.$ $1,59 ; 1,92)$ e por nutricionista $\left(\mathrm{RP}=1,80-\mathrm{IC}_{95 \%}\right.$ $1,64 ; 1,97)$. As equipes de saúde atuantes em UBS com estrutura adequada apresentaram frequência duas vezes maior de adequação do processo de trabalho $\left(\mathrm{RP}=2,09-\mathrm{IC}_{95 \%} 1,92 ; 2,28\right)$. 0 modelo ajustado diminuiu a presença do desfecho nas unidades apoiadas pelo NASF, tanto na adequação de estrutura $\left(\mathrm{RP}=1,03-\mathrm{IC}_{95 \%} 0,97 ; 1,10\right)$ como na adequação de processo de trabalho $(\mathrm{RP}=1,22-$ $\left.\mathrm{IC}_{95 \%} 1,07 ; 1,38\right)$. Nas demais variáveis, as análises ajustadas reduziram a ocorrência de ambos os desfechos em algumas categorias analisadas, porém mantiveram a direção da associação $(\mathrm{p}<0,001)$ (Tabela 2). 


\section{Discussão}

0 presente estudo encontrou baixas proporções de adequação da estrutura e processo de trabalho para as ações de alimentação e nutrição, em todas as unidades e equipes de saúde analisadas no âmbito da APS, no Brasil. Entre os limites da proposta metodológica, encontra-se a utilização de dados secundários dos sistemas de informações e de um programa de avaliação institucional, que podem apresentar divergências. 0 processo de conferência dos dados, assim como os critérios de inclusão de municípios que aderiram com a quase totalidade de suas equipes ao PMAQ, propõem-se a minimizar tais aspectos.

A utilização de dados do ano de 2014, igualmente, pode interferir, enquanto limite temporal da análise, diante da realidade atual, em função das mudanças que ocorreram nos aspectos de financiamento da APS, da reedição da Política Nacional de Atenção Básica em 2017 e da extinção dos repasses financeiros aos NASFs. No entanto, trata-se de registros de um panorama anterior a essas alterações.

Apesar de 35\% das UBS do país apresentarem estrutura adequada para as ações de alimentação e nutrição, menos de $8 \%$ das equipes de saúde realizam processo de trabalho adequado, tendo como base as ações selecionadas para este estudo. São as mesmas ações de promoção, prevenção e cuidados relacionados aos agravos nutricionais de maior magnitude previstas na 'Matriz de Ações de Alimentação e Nutrição na Atenção Básica de Saúde, publicada em 2009, ${ }^{13}$ e noutros documentos de responsabilidade do Ministério da Saúde, desde a edição da primeira versão da Política Nacional de Alimentação e Nutrição em 1999 - reformulada em 2012. ${ }^{8}$ Os resultados encontrados, portanto, indicam baixa adesão das equipes às recomendações normativas, associadas ou não a uma estrutura inadequada, e sua incorporação no processo de trabalho.

Entre os motivos para a baixa realização das ações de alimentação e nutrição pelas equipes de saúde - possivelmente associados -, encontra-se a falta de ações de educação permanente que incentivem a incorporação das diretrizes e protocolos atuais no processo de trabalho, a necessidade de medidas de gestão que induzam a realização dessas ações e, todavia a inexistência de processos visando ao seu monitoramento e avaliação. ${ }^{14}$

A estrutura e o processo de trabalho naAPS são objeto de diferentes estudos, enquanto fatores fundamentais para a qualidade da atenção e integralidade do cuidado, ${ }^{15,16}$ por conseguinte associados à redução de taxas de internação por condições sensíveis à atenção primária (ICSAP) $\cdot{ }^{17}$ No caso das ações de alimentação e nutrição, a baixa adequação de estrutura e processo de trabalho pode influenciar, negativamente, aspectos da vigilância alimentar e nutricional, com ênfase na precarização do acompanhamento do desenvolvimento infantil e investigação dos casos de desnutrição. ${ }^{18,19}$

Entre os itens analisados para a estrutura, insumos como sulfato ferroso, ácido fólico e sais para reidratação oral não estavam disponíveis em aproximadamente $10 \%$ das UBS. No Brasil, entre as carências de micronutrientes, a anemia ferropriva destaca-se por apresentar prevalência média de $53 \%$ das crianças até 5 anos, podendo atingir até $77,5 \%$ das crianças nessa faixa etária em localidades socialmente vulneráveis. ${ }^{20}$ A existência de programas de abrangência nacional para suplementação de ferro e ácido fólico data da implantação do SUS. Em 2005, instituiu-se o Programa Nacional de Suplementação de Ferro, atualizado em 2014, o que tornou o sulfato ferroso um medicamento obrigatório na Atenção Básica, e o incluiu na Relação Nacional de Medicamentos Essenciais (Rename), para ser ofertado, de forma profilática e universal, a crianças entre 6 e 24 meses, gestantes e mulheres até $03^{\circ}$ mês pós-parto e pós-aborto. ${ }^{21}$

Entretanto, as altas prevalências de condições crônicas, como a hipertensão arterial sistêmica, o diabetes mellitus e a obesidade, obrigam a uma vigilância continuada, para possibilitar a atuação preventiva sobre os agravos. ${ }^{22}$ Além de as unidades de saúde não apresentarem uma estrutura adequada à realização das ações de vigilância alimentar e nutricional (falta de balanças, réguas antropométricas etc.), o registro de dados nos sistemas de informações não é realizado por todas as equipes de saúde quando, em detrimento dessas ações, priorizam a mensuração de peso e altura de gestantes e crianças menores de 2 anos. Quase 30\% das equipes de APS não realizam ações de promoção da alimentação saudável. Este cenário corrobora o descrito em estudos que indicam as baixas coberturas do Sistema de Vigilância Alimentar e Nutricional (SISVAN), no que se refere ao monitoramento do estado nutricional da população local. ${ }^{23-25}$ Consequentemente, o sistema é subutilizado enquanto ferramenta de planejamento e gestão das ações de alimentação e nutrição. ${ }^{10}$ 
A estrutura das UBS e o processo de trabalho das equipes apresentaram maior adequação nos municípios de grande porte populacional. Diferentes estudos já associaram a estes municípios melhores serviços de APS, organizados sobre redes de saúde mais abrangentes, dotados com maiores investimentos e diversidade nos serviços ofertados, implicando maior capacidade de manutenção e sustentabilidade. ${ }^{13,26,27}$ Um fator que poderia interferir nesse achado seria a adesão espontânea, além do fato de os municípios maiores poderem selecionar as melhores equipes para participar de um programa de avaliação, o que não seria possível em municípios de menor porte e número reduzido de equipes. Esse viés foi minimizado pela estratégia metodológica do estudo: incluir na análise UBS e equipes localizadas em munícipios com adesão de pelo menos $80 \%$ das equipes.

Equipes e unidades de saúde que contavam com o apoio do nutricionista e dos NASFs também apresentaram maior adequação de processo de trabalho. A presença de outros profissionais na APS, a partir da implantação dos NASFs tem estimulado o trabalho interdisciplinar e, com isso, a possibilidade de ações serem desenvolvidas por diferentes profissionais na APS. ${ }^{28}$ As atividades de apoio matricial contribuem para a ampliação do acesso da população aos cuidados em saúde, e são baseadas no princípio da educação permanente: por exemplo, as atividades de apoio à gestão do cuidado clínico e do processo de trabalho, além das intervenções no território ${ }^{28}$ Vale destacar que, embora a presença do nutricionista tenha interferido nos resultados, as ações analisadas são aquelas que, como mínimo, fariam parte da rotina da Atenção Básica e não dependeriam, necessariamente, da presença desse profissional. 0 fato aponta não só para a oportunidade, mas também para a necessidade da qualificação dos profissionais da APS na orientação à população sobre alimentação adequada e saudável, como parte do processo de trabalho em saúde. ${ }^{29}$

A despeito da baixa prevalência de adequação do processo de trabalho das equipes de saúde no Brasil, a análise concluiu que a presença de estrutura adequada das UBS para as ações de alimentação e nutrição impacta positivamente na adequação do processo de trabalho das equipes que nelas atuam. É evidente que, para o melhor desenvolvimento das ações de alimentação e nutrição no Brasil, são necessários maiores investimentos em estruturação das UBS, além de qualificação das equipes no enfoque das estratégias de promoção, prevenção e controle de agravos à saúde. Outros estudos, a analisarem estrutura e processo de trabalho para diferentes ações temáticas na APS, também concluíram que há necessidade de investimentos na estruturação dos serviços; e consideraram a possibilidade de utilização dos recursos do próprio PMAQ-AB nessas melhorias. ${ }^{13,27}$

A adequação de processo de trabalho foi maior entre as equipes que não possuem população descoberta em sua área de abrangência. Trata-se de um indicativo de que a capacidade de cobertura das UBS, diante da demanda populacional, pode interferir na adequação de suas equipes para o desempenho das ações programadas.

As crescentes demandas de acesso à APS, a escassez de recursos destinados a esse nível de atenção, assim como a necessidade do cumprimento de metas por parte das equipes de saúde, podem ter influenciado os achados do estudo. A Atenção Primária à Saúde, todavia, mantém e reforça a priorização de atendimento a determinados ciclos de vida, característica de modelos fragmentados de atenção à saúde, frente às necessidades de acesso universal. Avanços visando romper essa lógica programática são fundamentais para a qualificação do processo de trabalho e, consequentemente, a integralidade da atenção à saúde.

\section{Contribuições das autoras}

Machado PMO e Lacerda JT contribuíram na concepção e delineamento do estudo, análise e interpretação dos resultados, redação e revisão crítica do conteúdo do manuscrito. Colussi CF e Calvo MCM contribuíram na análise e interpretação dos dados, redação e revisão crítica do conteúdo do manuscrito. Todas as autoras aprovaram a versão final do manuscrito e são responsáveis por todos os seus aspectos, incluindo a garantia de sua precisão e integridade. 


\section{Referências}

1. Brasil, Ministério da Saúde, Secretaria de Vigilância em Saúde, Departamento de Análise de Situação de Saúde. Plano de ações estratégicas para o enfrentamento das doenças crônicas não transmissíveis (DCNT) no Brasil 2011-2022. Brasília, DF: Ministério da Saúde; 2011.

2. Malta DC, Bernal RTI, Nunes ML, Oliveira MM, Iser BPM, Andrade SSCA, et al. Prevalência de fatores de risco e proteção para doenças crônicas não transmissíveis em adultos: estudo transversal, Brasil 2012. Epidemiol Serv Saude. 2014;23(4):609-22. doi: 10.5123/S1679-49742014000400003.

3. Batista Filho B, Rissin A. A transição nutricional no Brasil: tendências regionais e temporais. Cad Saude Publica. 2003;19(Suppl 1):S181-S91. doi: 10.1590/ S0102-311X2003000700019. Epub 2003 Jul 21.

4. Coutinho JG, Gentil PC, Toral N. A desnutrição e obesidade no Brasil: o enfrentamento com base na agenda única da nutrição. Cad Saude Publica. 2008;24(Suppl 2):S332-S340. doi: 10.1590/S0102311X2008001400018.

5. Hearn L, Miller M, Cross D. Engaging primary health care providers in the promotion of healthy weight among young children: barriers and enablers for policy and management. Saúde Aust J Prim. 2007;13(2):66-79. doi: 10.1071/PY07025.

6. Phuka J, Maleta K, Thomas M, Gladstone M. A job analysis of community health workers in the context of integrated nutrition and early child development. Ann N Y Acad of Sci. 2014 Jan;1308:183-91. doi: 10.1111/nyas.12338.

7. Jaime PC, Silva ACF, Lima AMC, Bortolini GA. Ações de alimentação e nutrição na atenção básica: a experiência de organização no governo brasileiro. Rev Nutr. 2011;24(6):809-24. doi: 10.1590/\$141552732011000600002 .

8. Brasil, Ministério da Saúde. Política Nacional de Alimentação e Nutrição. Brasília, DF: Ministério da Saúde; 2012.

9. Ramos MKP, Lima AMC, Gubert MB. Agenda para intensificação da atenção nutricional à desnutrição infantil: resultados de uma pactuação interfederativa no Sistema Único de Saúde. Rev Nutr. 2015;28(6):641-53. doi: 10.1590/141552732015000600007.

10. Rolim MD, Lima SML, Barros DC, Andrade CLT. Avaliação do SISVAN na gestão de ações de alimentação e nutrição em Minas Gerais, Brasil.
Cienc Saude Colet. 2015;20(8):2359-69. doi: 10.1590/1413-81232015208.00902015.

11. Brasil, Ministério da Saúde. Programa nacional de melhoria do acesso e da qualidade da atenção básica (PMAQ) 2018 [citado 22 jan. 2021]. Disponível em: https://aps.saude.gov.br/ape/pmaq

12. Barros AJD, Hirakata VN. Alternatives for logistic regression in cross-sectional studies: an empirical comparison of models that directly estimate the prevalence ratio. BMC Med Res Methodol. 200 Oct 20;3:21. doi: 10.1186/1471-2288-3-21.

13. Brasil, Ministério da Saúde, Secretaria de Atenção à Saúde, Departamento de Atenção Básica. Matriz de ações de alimentação e nutrição na atenção básica de saúde. Brasília, DF: Ministério da Saúde; 2009.

14. Jaime PC, Santos LMP. Transição nutricional e a organização do cuidado em alimentação e nutrição na Atenção Básica em saúde. Divulg saúde debate. 2014 [citado 22 jan. 2021];51:72-85. Disponível em: http://cebes.org.br/site/wp-content/uploads/2014/12/ Divulgaca0-51.pdf.

15. omasi E, Oliveira TF, Fernandes PAA, Thumé E, Silveira DS, Siqueira FV, et al. Estrutura e processo de trabalho na prevenção do câncer de colo de útero na Atenção Básica à Saúde no Brasil: Programa de Melhoria do Acesso e da Qualidade - PMAQ. Rev Bras Saude Mater Infant. 2015;15(2):171-80. doi: 10.1590/\$1519-38292015000200003.

16. Rocha ACD, Sousa CPC, Queiroz D, Figueroa Pedraza D. Atenção básica à saúde: avaliação de estrutura e processo. RAS. 2012;14(54):71-9. Disponível em: http://www.cqh.org.br/portal/pag/anexos/baixar. php?p_ndoc $=276 \&$ p_nanexo $=321$.

17. Araujo WRM, Queiroz RCS, Rocha TAH, Silva NCD, Thumé E, Tomasi E, et al. Structure and work process in primary care and hospitalizations for sensitive conditions. Rev Saude Publica. 2017 Aug 17;51:75. doi: 10.11606/S1518-8787.2017051007033.

18. Rocha ACD, Figueroa Pedraza D. Acompanhamento do crescimento infantil em unidades básicas de saúde da família do município de Queimadas, Paraíba, Brasil. Tex Context Enfermagem. 2013;22(4):116978. doi: 10.1590/S0104-07072013000400036.

19. Enes CC, Loiola H, Oliveira MR. Cobertura populacional do Sistema de Vigilância Alimentar e Nutricional no Estado de São Paulo, Brasil. Cienc Saude Colet. 2014;19(5):1543-51. doi: 10.1590/1413-81232014195.05872013. 
20. Jordão RE, Bernardi JLD, Barros Filho AA. Prevalência de anemia ferropriva no Brasil: uma revisão sistemática. Rev Paul Pediatr. 2009;27(1):90-8. doi: 10.1590/S0103-05822009000100014.

21. Ministério da Saúde (BR). Portaria $n^{0} 1.977$, de 12 de setembro de 2014 . Atualiza as diretrizes nacionais do Programa Nacional de Suplementação de Ferro (PNSF) da Política Nacional de Alimentação e Nutrição (PNAN). Brasília, DF: Diário Oficial da União; 2014 [citado 22 jan. 2021]. Disponível em: http://bvsms.saude.gov.br/bvs/saudelegis/gm/2014/ prt1977_12_09_2014.html.

22. Malta DC, Moraes Neto OL, Silva Junior JB. Apresentação do plano de ações estratégicas para o enfrentamento das doenças crônicas não transmissíveis no Brasil, 2011 a 2022. Epidemiol Serv Saude. 2011;20(4):425-38. doi: 10.5123/\$167949742011000400002

23. Burlandy L. Avaliação nutricional na atenção básica à luz dos princípios que fundamentam a organização da atenção nutricional no Sistema Único de Saúde. Cad Saude Publica. 2019;35(11):e00155719. doi:10.1590/0102-311x00155719.

24. Jung NM, Bairros FS, Neutzling MB. Utilização e cobertura do Sistema de Vigilância Alimentar e Nutricional no Estado do Rio Grande do Sul, Brasil. Cienc Saude Colet. 2014;19(5):1379-88. doi: 10.1590/1413-81232014195.20002013.
25. Canella DS, Silva ACF, Jaime PC. Produção científica sobre nutrição no âmbito da Atenção Primária à Saúde no Brasil: uma revisão de literatura. Cienc Saude Colet. 2013;18(2):297-308. doi: 10.1590/ S1413-81232013000200002.

26. Calvo MCM, Lacerda JTd, Colussi CF, Schneider IJC, Rocha TAH. Estratificação de municípios brasileiros para avaliação de desempenho em saúde. Epidemiol Serv Saude. 2016;25(4):767-776. doi: 10.5123/ S1679-49742016000400010.

27. Neves RG, Duro SMS, Muñiz J, Castro TRP, Facchini LA, Tomasi E. Estrutura das unidades básicas de saúde para atenção às pessoas com diabetes: Ciclos I e II do Programa Nacional de Melhoria do Acesso e da Qualidade. Cad Saude Publica. 2018;34(4):e00072317. doi: 10.1590/0102$311 X 00072317$.

28. Fittipaldi ALM, Barros DC, Romano VF. Apoio matricial nas ações de alimentação e nutrição: visão dos profissionais da Estratégia Saúde da Família. Physis. 2017;27(3):793-811. doi: 10.1590/s010373312017000300021 .

29. Bortolini GA, Oliveira TFV, Silva SA, Santin RC, Medeiros OL, Spaniol AM, et al. Ações de alimentação e nutrição na atenção primária à saúde no Brasil. Rev Panam Salud Publica. 2020;44:e39. doi:10.26633/ RPSP.2020.39 


\section{Abstract}

Objective: To evaluate the conditions of structure and work process for food and nutrition actions in Primary Health Care in Brazil. Methods: This was a cross-sectional study with secondary data from the Program for Primary Health Care Access and Quality Improvement. The proportions of Primary Health Care Center (PHCC) and health team adequacy were described according to organizational variables, using prevalence ratios and Poisson regression. Results: 19,793 PHCCs and 24,549 teams were analyzed; $35.0 \%(n=6,928)$ of PHCCs were considered adequate in relation to structure and $7.9 \%(n=1,934)$ of the teams had adequate work process. Standing out in the analyses of association are PHCCs in the Southern region (44.7\%) and teams in the Southeastern region (10.9\%), in addition to municipalities with more than 300,000 inhabitants. Conclusion: The PHCCs and teams analyzed presented a low proportion of adequacy for structure and work process for food and nutrition actions.

Keywords: Structure of Services; Primary Health Care; Nutrition Programs and Policies; Cross-Sectional Studies.

\section{Resumen}

Objetivo: Evaluar las condiciones de estructura $y$ proceso de trabajo de las acciones de alimentación y nutrición en la Atención Primaria de Salud en Brasil. Métodos: Estudio transversal con datos del Programa de Mejoramiento del Acceso y la Calidad en la Atención Primaria. Se describieron las proporciones de adecuación de las Unidades Básicas de Salud (UBS) y equipos de salud según variables organizacionales por la razón de prevalencia y regresión de Poisson. Resultados: Se analizaron 19.793 UBS y 24.549 equipos. El 35,0\% ( $n=6.928)$ de las UBS se consideraron adecuadas en la estructuray el 7,9\% ( $n=1.934)$ de los equipos adecuados al proceso de trabajo. En los análisis asociativos se destacaron las UBS de la región Sur (44,7\%) y los equipos de la región Sudeste (10,9\%), además de los municipios con más de 300 mil habitantes. Conclusión: Las UBS y los equipos analizados presentaron bajas proporciones de adecuación para la estructura y el proceso de trabajo para las acciones de alimentación y nutrición.

Palabras clave: Estructura de los Servicios; Atención Primaria de Salud; Programas y Políticas de Nutrición y Alimentación; Estudios Transversales.

Recebido em 24/08/2020

Aprovado em 04/12/2020

Editora associada: Doroteia Aparecida Höfelmann - (1) orcid.org/0000-0003-1046-3319 